\title{
A Low Complexity Scheme for Transmit Diversity Over Frequency Selective Channels
}

\author{
Pei Xiao, Mathini Sellathurai, Tharm Ratnarajah \\ The Institute of Electronics, Communications and Information Technology \\ Queen's University Belfast, BT3 9DT, United Kingdom \\ E-mail: pei.xiao,m.sellathurai,t.ratnarajah@ecit.qub.ac.uk
}

\begin{abstract}
A low complexity transmit diversity scheme is derived in this paper in order to overcome the prohibitive complexity imposed by the maximum likelihood detection for the systems with space-time block code (STBC) over frequency selective channels. By taking advantage of multipath propagation and exploiting temporal diversity gain, the proposed turbo equalization algorithm significantly improves the system performance compared to the original Alamouti algorithm as well as the conventional minimum mean square error (MMSE) detection scheme.
\end{abstract}

\section{INTRODUCTION}

In recent years, space-time coding has emerged as one of the most promising technologies for meeting the high data rate and high service quality requirements. There are two main types of space-time codes, namely, space-time trellis codes (STTC) [1] and space-time block codes (STBC) [2]. The advantage of STTCs over STBCs is the provision of coding gain. However, this is achieved at the cost of high complexity encoders and decoders. For the purpose of this work, we consider the use of STBC, in particular, the two-antenna transmit diversity scheme [2] proposed by Alamouti.

At high data rates, the intersymbol interference (ISI) introduced by frequency-selective fading channels becomes a severe problem. The Alamouti algorithm was originally developed for flat fading channels and so does not take into consideration the ISI introduced by frequency-selective fading channels. The key building block in combating ISI is the equalizer, or more effectively, the turbo equalizer that performs equalization and channel decoding jointly in an iterative manner. In [3], [4], the linear MMSE-filter based turbo equalization is proposed for single-input, single-output (SISO) channels, and is extended to multiple-input, multiple-output (MIMO) systems, e.g., in [5][7]. However, the MMSE equalizers introduced in [5], [6] are applied to the MIMO systems without STBC or STTC. Moreover, only a single-input, multiple-output system (for a single user) is considered in [6]. The turbo multiuser detection technique previously proposed in [3] is extended to STBC and STTC coded systems in [7]. However, the studied case is only confined to the flat-fading channels. The main contribution of this paper is the proposal of a turbo equalization scheme based on a different approach, which is well suited for combating ISI introduced by the frequency selective MIMO channels and in the meantime, achieving spatial and temporal diversities. First, we derive the maximum likelihood detection algorithm for the systems under question. Its unaffordable computational complexity calls for suboptimum solutions. To this end, we introduce a low complexity scheme which is shown to achieve significant performance gain compared to the original Alam- outi algorihtm and the conventional MMSE filter based turbo equalization for the STBC coded systems.

\section{SySTEM MODEL}

Fig. 1 shows the baseband representation of the two branch transmit diversity scheme under study. The information sequence $\left\{b_{n}\right\}$ is convolutionally encoded into coded bits $\left\{u_{n}\right\}$, which are subsequently interleaved and each block of two coded and interleaved bits $u_{n}^{\prime}[0], u_{n}^{\prime}[1]$ is mapped into one of the four QPSK symbols $s_{n}$. The interleaver and deinterleaver are denoted as $\Pi$ and $\Pi^{-1}$, in Fig. 1 and 2, respectively. We use the space-time coding scheme proposed in [2]. The transmitted symbols are grouped into blocks of 2 symbols at each antenna. At a given time, two symbols are simultaneously transmitted from two antennas. At time instance $t$, the symbol transmitted from the first antenna is denoted as $s_{n}^{0}$, and the symbol transmitted from the second antenna is denoted as $s_{n}^{1}$. During the next symbol period $t+T$, symbol $-s_{n}^{1 *}$ is transmitted from the first antenna, and $s_{n}^{0 *}$ is transmitted from the second antenna, where ${ }^{*}$ denotes the conjugate transpose operation when applied to vectors and matrices, and simply the complex conjugate when applied to scalars.

For simplicity, we assume two transmit antennas, one receive antenna and each channel has 3 taps in the derivation of the proposed turbo equalization algorithm. However, its extension to systems with multiple receive antennas and generic ISI channels is straightforward. Each complex channel coefficient is denoted as $h_{i j}^{l}$ where the first (second) subscript $i(j)$ is the index of the transmit (receive) antenna, the superscript $l$ refers to the number of the channel tap. The received signals at antenna rx0 during the two symbol periods $t$ and $t+T$ can be formed as

$r_{n}^{0}=h_{00}^{2} s_{n-1}^{0}-h_{00}^{1} s_{n-1}^{1 *}+\underline{h_{00}^{0} s_{n}^{0}}+h_{10}^{2} s_{n-1}^{1}+h_{10}^{1} s_{n-1}^{0 *}+\underline{h_{10}^{0} s_{n}^{1}}+w_{n}^{0}$
$r_{n}^{1}=-h_{00}^{2} s_{n-1}^{1 *}+h_{00}^{1} s_{n}^{0}-\underline{h_{00}^{0} s_{n}^{1 *}}+h_{10}^{2} s_{n-1}^{0 *}+h_{10}^{1} s_{n}^{1}+\underline{h_{10}^{0} s_{n}^{0 *}+w_{n}^{1}}$

where $w_{n}^{0}, w_{n}^{1}$ are the complex additive white Gaussian noise with zero mean and variance $N_{0}$. The desired symbols in the previous equations are underlined so that they can be distinguished from the interference symbols and the noise.

\section{A Low Complexity Turbo Equalizer}

The task of the receiver is to detect the transmitted information bits $\left\{b_{n}\right\}$ given the received observations $\left\{r_{n}^{0}, r_{n}^{1}\right\}$. To this end, we need first to detect the transmitted QPSK symbols $\left\{s_{n}^{0}, s_{n}^{1}\right\}$ which are corrupted with ISI and AWGN. The estimated symbols are then converted to coded bits, which are subsequently deinterleaved and decoded to obtain an estimate of the information sequence. For the received signals 


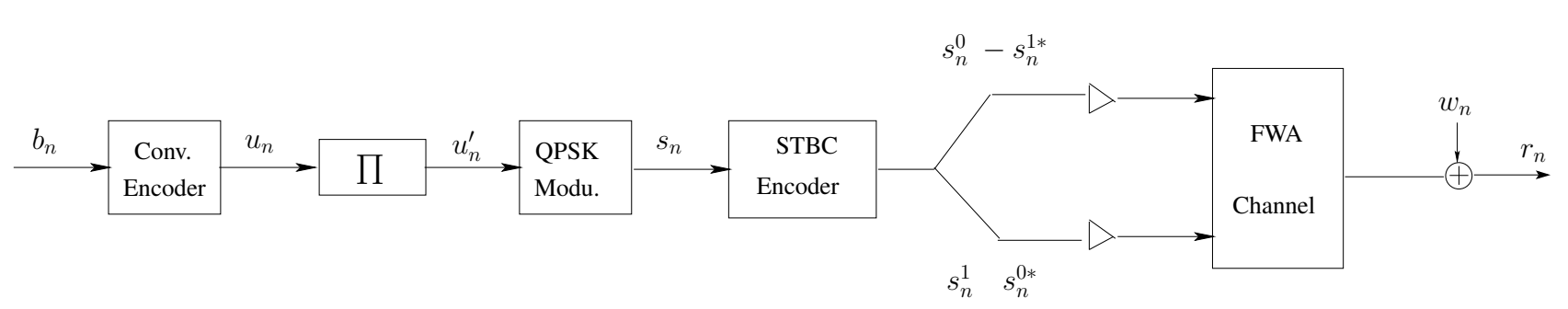

Fig. 1. Diagram for the STBC coded system with 2TX-1RX antennas.

expressed by (1), a direct implementation of the Alamouti algorithm yields

$$
\begin{aligned}
& \widetilde{s_{n}^{0}}=\hat{h}_{00}^{0 *} r_{n}^{0}+\hat{h}_{10}^{0} r_{n}^{1 *}=\left(\hat{h}_{00}^{0 *} h_{00}^{0}+\hat{h}_{10}^{0 *} h_{10}^{0}\right) s_{n}^{0}+\eta_{n}^{0} ; \\
& \widetilde{s_{n}^{1}}=\hat{h}_{10}^{0 *} r_{n}^{0}-\hat{h}_{00}^{0} r_{n}^{1 *}=\left(\hat{h}_{00}^{0 *} h_{00}^{0}+\hat{h}_{10}^{0 *} h_{10}^{0}\right) s_{n}^{1}+\eta_{n}^{1},
\end{aligned}
$$

where $\hat{h}_{i j}^{l}$ denotes the estimate of $h_{i j}^{l}, \eta_{n}^{0}, \eta_{n}^{1}$ denote the combined ISI and noise, e.g., $\eta_{n}^{0}=\hat{h}_{00}^{0 *}\left(h_{10}^{0} s_{n}^{1}+h_{00}^{2} s_{n-1}^{0}-\right.$ $\left.h_{00}^{1} s_{n-1}^{1 *}+h_{10}^{2} s_{n-1}^{1}+h_{10}^{1} s_{n-1}^{0 *}+w_{n}^{0}\right)+\hat{h}_{10}^{0}\left(-h_{00}^{0 *} s_{n}^{1}-h_{00}^{2 *} s_{n-1}^{1}+\right.$ $\left.h_{00}^{1 *} s_{n}^{0 *}+h_{10}^{2 *} s_{n-1}^{0}+h_{10}^{1 *} s_{n}^{1 *}+w_{n}^{1 *}\right)$.

The Alamouti algorithm achieves maximum likelihood (ML) detection for flat fading channels. However, it does not take into consideration the ISI introduced by frequency-selective fading channels. It is apparent to see from (2) that a large number of ISI terms would inevitably lead to an error floor in the BER performance. In order to derive an optimum detection algorithm (in the sense of maximum likelihood) for the system under question, we need to reform the received signal expressed in (1) in vector form as

$$
\mathbf{r}_{n}=\mathbf{S}_{n} \mathbf{h}+\mathbf{w}_{n},
$$

where

$$
\begin{aligned}
\mathbf{r}_{n} & =\left[\begin{array}{lllll}
r_{n}^{0} & r_{n}^{1} & r_{n+1}^{0} & r_{n+1}^{1}
\end{array}\right]^{T} ; \\
\mathbf{w}_{n} & =\left[\begin{array}{llllll}
w_{n}^{0} & w_{n}^{1} & w_{n+1}^{0} & w_{n+1}^{1}
\end{array}\right]^{T} ; \\
\mathbf{h} & =\left[\begin{array}{llllll}
h_{00}^{0} & h_{00}^{1} & h_{00}^{2} & h_{10}^{0} & h_{10}^{1} & h_{10}^{2}
\end{array}\right]^{T} ; \\
\mathbf{S}_{n} & =\left[\begin{array}{cccccc}
s_{n}^{0} & -s_{n-1}^{1 *} & s_{n-1}^{0} & s_{n}^{1} & s_{n-1}^{0 *} & s_{n-1}^{1} \\
-s_{n}^{1 *} & s_{n}^{0} & -s_{n-1}^{1 *} & s_{n}^{0 *} & s_{n}^{1} & s_{n-1}^{0 *} \\
s_{n+1}^{0} & -s_{n}^{1 *} & s_{n}^{0} & s_{n+1}^{1} & s_{n}^{0 *} & s_{n}^{1} \\
-s_{n+1}^{1 *} & s_{n+1}^{0} & -s_{n}^{1 *} & s_{n+1}^{0 *} & s_{n+1}^{1} & s_{n}^{0 *}
\end{array}\right],
\end{aligned}
$$

where ${ }^{T}$ denotes the transpose operation. For generic channels, the channel vector $\mathbf{h}$ is a $2 L$-element vector, and the matrix $\mathbf{S}_{n}$ is a $4 \times 2 L$ matrix, where $L$ is the number of channel taps. Since the noise vector $\mathbf{w}_{n}$ is complex Gaussian, the likelihood function and log-likelihood function of the received vector $\mathbf{r}_{n}$ conditioned on a realization of the fading channels and transmitted data can be expressed as

$$
\begin{aligned}
p\left(\mathbf{r}_{n}\right) & =\frac{1}{\left(\pi N_{0}\right)^{4}} \exp \left(-\frac{\left\|\mathbf{r}_{n}-\mathbf{S}_{n} \mathbf{h}\right\|^{2}}{N_{0}}\right) ; \\
\ln p\left(\mathbf{r}_{n}\right) & =\text { constant }-\frac{1}{N_{0}}\left\|\mathbf{r}_{n}-\mathbf{S}_{n} \mathbf{h}\right\|^{2} .
\end{aligned}
$$

From (4), one can see that maximization of this loglikelihood function is equivalent to minimization of the function $\left\|\mathbf{r}_{n}-\mathbf{S}_{n} \mathbf{h}\right\|^{2}$. This algorithm needs the estimate of the whole matrix $\mathbf{S}_{n}$ which is a function of $s_{n-1}^{0}, s_{n-1}^{1}, s_{n}^{0}, s_{n}^{1}, s_{n+1}^{0}, s_{n+1}^{1}$. For QPSK modulation, it involves a testing of $4^{6}$ hypotheses for the 3-tap channel. In general, the possible number combinations for forming the matrix $\mathbf{S}_{n}$ is $M^{2 L}$, where $M$ is the constellation size and
$L$ is the number of channel taps. Apparently, the complexity of this ML detection grows exponentially with $L$. Next, we propose a sub-optimum solution to reduce the complexity from exponential to polynomial. The idea is to use the original Alamouti algorithm or its modified version at the initial stage to get a rough estimate of the transmitted data. Then the algorithm switches to the decision directed mode. Let us reform the matrix $\mathbf{S}_{n}$ as

$$
\mathbf{S}_{n}=\mathbf{S}_{n}^{0}+\mathbf{s}_{n}^{0} \approx \tilde{\mathbf{S}}_{n}^{0}+\mathbf{s}_{n}^{0} ; \quad \mathbf{S}_{n}=\mathbf{S}_{n}^{1}+\mathbf{s}_{n}^{1} \approx \tilde{\mathbf{S}}_{n}^{1}+\mathbf{s}_{n}^{1}
$$

and

$$
\begin{aligned}
& \tilde{\mathbf{S}}_{n}^{0}=\left[\begin{array}{cccccc}
0 & -\hat{s}_{n-1}^{1 *} & \hat{s}_{n-1}^{0} & \hat{s}_{n}^{1} & \hat{s}_{n-1}^{0 *} & \hat{s}_{n-1}^{1} \\
-\hat{s}_{n}^{1 *} & 0 & -\hat{s}_{n-1}^{1 *} & 0 & \hat{s}_{n}^{1} & \hat{s}_{n-1}^{0 *} \\
\hat{s}_{n+1}^{0} & -\hat{s}_{n}^{1 *} & 0 & \hat{s}_{n+1}^{1} & 0 & \hat{s}_{n}^{1} \\
-\hat{s}_{n+1}^{1 *} & \hat{s}_{n+1}^{0} & -\hat{s}_{n}^{1 *} & \hat{s}_{n+1}^{0 *} & \hat{s}_{n+1}^{1} & 0
\end{array}\right] ; \\
& \mathbf{s}_{n}^{0}=\left[\begin{array}{cccccc}
s_{n}^{0} & 0 & 0 & 0 & 0 & 0 \\
0 & s_{n}^{0} & 0 & s_{n}^{0 *} & 0 & 0 \\
0 & 0 & s_{n}^{0} & 0 & s_{n}^{0 *} & 0 \\
0 & 0 & 0 & 0 & 0 & s_{n}^{0 *}
\end{array}\right] \\
& \tilde{\mathbf{S}}_{n}^{1}=\left[\begin{array}{cccccc}
\hat{s}_{n}^{0} & -\hat{s}_{n-1}^{1 *} & \hat{s}_{n-1}^{0} & 0 & \hat{s}_{n-1}^{0 *} & \hat{s}_{n-1}^{1} \\
0 & \hat{s}_{n}^{0} & -\hat{s}_{n-1}^{1 *} & \hat{s}_{n}^{0 *} & 0 & \hat{s}_{n-1}^{0 *} \\
\hat{s}_{n+1}^{0} & 0 & \hat{s}_{n}^{0} & \hat{s}_{n+1}^{1} & \hat{s}_{n}^{0 *} & 0 \\
-\hat{s}_{n+1}^{1 *} & \hat{s}_{n+1}^{0} & 0 & \hat{s}_{n+1}^{0 *} & \hat{s}_{n+1}^{1} & \hat{s}_{n}^{0 *}
\end{array}\right] \\
& \mathbf{s}_{n}^{1}=\left[\begin{array}{cccccc}
0 & 0 & 0 & s_{n}^{1} & 0 & 0 \\
-s_{n}^{1 *} & 0 & 0 & 0 & s_{n}^{1} & 0 \\
0 & -s_{n}^{1 *} & 0 & 0 & 0 & s_{n}^{1} \\
0 & 0 & -s_{n}^{1 *} & 0 & 0 & 0
\end{array}\right]
\end{aligned}
$$

where $\hat{s}_{n+i}$ is an estimate of $s_{n+i}$ from previous iteration, and $\tilde{\mathbf{S}}_{n}^{0}\left(\tilde{\mathbf{S}}_{n}^{1}\right)$ is an estimate of $\mathbf{S}_{n}^{0}\left(\mathbf{S}_{n}^{1}\right)$, the elements of which are symbol estimates from previous iteration. The key to achieve complexity reduction is to split the matrix $\mathbf{S}_{n}$ into two matrices, the first one $\tilde{\mathbf{S}}_{n}^{0}\left(\tilde{\mathbf{S}}_{n}^{1}\right)$ contains the interference symbols which are fixed with the symbol estimates from previous iteration; whereas the second matrix $\mathbf{s}_{n}^{0}\left(\mathbf{s}_{n}^{1}\right)$ only contains the desired symbol to be detected. Therefore, only 4 (or $M$ in general) hypotheses need to be tested instead of $4^{6}$ (or $M^{2 L}$ in general) as in the case of the original $\mathrm{ML}$ algorithm. The complexity is thus drastically reduced. With the matrix decomposition, the function $\left\|\mathbf{r}_{n}-\mathbf{S}_{n} \mathbf{h}\right\|^{2}$ can be expanded as

$$
\begin{aligned}
& \left\|\mathbf{r}_{n}-\mathbf{S}_{n} \mathbf{h}\right\|^{2}=\left\|\mathbf{r}_{n}-\tilde{\mathbf{S}}_{n}^{0} \mathbf{h}-\mathbf{s}_{n}^{0} \mathbf{h}\right\|^{2} \\
& =\left\|\mathbf{r}_{n}-\tilde{\mathbf{S}}_{n}^{0} \mathbf{h}\right\|^{2}-2 \operatorname{Re}\left\{\left(\mathbf{s}_{n}^{0} \mathbf{h}\right)^{*}\left[\mathbf{r}_{n}-\tilde{\mathbf{S}}_{n}^{0} \mathbf{h}\right]\right\}+\left\|\mathbf{s}_{n}^{0} \mathbf{h}\right\|^{2} .
\end{aligned}
$$

Since the first term of the previous expression is irrelevant to the choice of $s_{n}^{0}$, the proposed algorithm can thus be expressed as

$$
\hat{s}_{n}^{0}=\arg \max _{s_{q}^{0} \in\left\{s_{0}, s_{1}, s_{2}, s_{3}\right\}} 2 \operatorname{Re}\left\{\left(\mathbf{s}_{q}^{0} \mathbf{h}\right)^{*}\left[\mathbf{r}_{n}-\tilde{\mathbf{S}}_{n}^{0} \mathbf{h}\right]\right\}-\left\|\mathbf{s}_{q}^{0} \mathbf{h}\right\|^{2} .
$$


Similarly,

$$
\hat{s}_{n}^{1}=\arg \max _{s_{q}^{1} \in\left\{s_{0}, s_{1}, s_{2}, s_{3}\right\}} 2 \operatorname{Re}\left\{\left(\mathbf{s}_{q}^{1} \mathbf{h}\right)^{*}\left[\mathbf{r}_{n}-\tilde{\mathbf{S}}_{n}^{1} \mathbf{h}\right]\right\}-\left\|\mathbf{s}_{q}^{1} \mathbf{h}\right\|^{2},
$$

where

$$
\begin{aligned}
& \mathbf{s}_{q}^{0} \mathbf{h}=\left[\begin{array}{llll}
s_{q}^{0} h_{00}^{0} & s_{q}^{0} h_{00}^{1}+s_{q}^{0 *} h_{10}^{0} & s_{q}^{0} h_{00}^{2}+s_{q}^{0 *} h_{10}^{1} & s_{q}^{0 *} h_{10}^{2}
\end{array}\right]^{T} ; \\
& \mathbf{s}_{q}^{1} \mathbf{h}=\left[\begin{array}{llll}
s_{q}^{1} h_{10}^{0} & s_{q}^{1} h_{10}^{1}-s_{q}^{1 *} h_{00}^{0} & s_{q}^{1} h_{10}^{2}-s_{q}^{1 *} h_{00}^{1} & -s_{q}^{1 *} h_{00}^{2}
\end{array}\right]^{T} .
\end{aligned}
$$

From (7) and (8), we see that the channel vector $\mathbf{h}$ has to be known or estimated in order to carry out the detection. The channel state information (CSI) can be obtained, e.g., by the channel estimation algorithms presented in [8].

In this paper, we are mainly concerned about the turbo equalization which combines data detection and channel decoding in an iterative fashion. Fig. 2 shows the proposed turbo equalization algorithm. First, the Alamouti algorithm is used to obtain an initial estimate of the transmitted symbols. The soft values of the transmitted symbols are computed in the form of a priori log-likelihood ratio (LLR) $\left\{\lambda\left(s_{n} ; I\right)=\right.$ $\left.\lambda\left(x_{n}\right)+j \lambda\left(y_{n}\right)\right\}$ where $s_{n}$ denotes either $s_{n}^{0}$ or $s_{n}^{1}$. The soft symbol estimates $\left\{\lambda\left(s_{n} ; I\right)\right\}$ are passed to the equalizer, which computes $\left\{\lambda\left(s_{n} ; O\right)\right\}$, the extrinsic information of $\left\{s_{n}\right\}$. A symbol-to-bit converter (SBC) maps $\left\{\lambda\left(s_{n} ; O\right)\right\}$ to the LLR values of coded bits $\left\{\lambda\left(u_{n}^{\prime} ; O\right)\right\}$, which are deinterleaved to yield $\left\{\lambda\left(u_{n} ; I\right)\right\}$. Based on the soft inputs $\left\{\lambda\left(u_{n} ; I\right)\right\}$, a LogMAP decoder computes the LLR for each information bit $\lambda\left(b_{n} ; O\right)$ and each coded bit $\lambda\left(u_{n} ; O\right)$. The former is used to make decisions on the transmitted information bit at the final iteration, and the latter is interleaved and passed through a bitto-symbol converter (BSC) to derive a soft symbol estimate $\lambda\left(s_{n} ; I\right)$, which is used for equalization at the next iteration, i.e., in order to carry out the operations expressed in (7) and (8) using soft decision feedback. To tackle the problem of error propagation, we can replace the hard decisions $\hat{s}_{n+i}$ in the interference matrix $\tilde{\mathbf{S}}_{n}^{0}\left(\tilde{\mathbf{S}}_{n}^{1}\right)$ in (6) with their soft estimates $\bar{s}_{n+i}=\bar{x}_{n+i}+j \bar{y}_{n+i}=\tanh \left[\lambda\left(x_{n+i}\right) / 2\right] / \sqrt{2}+$ $j \tanh \left[\lambda\left(y_{n+i}\right) / 2\right] / \sqrt{2}$, where $\lambda\left(x_{n+i}\right), \lambda\left(y_{n+i}\right)$ are the real and imaginary part of $\lambda\left(s_{n+i} ; I\right)$, respectively.

Next, we shall describe how the soft values can be derived at the output of the equalizer. From (7) and (8), we know that the $q^{\text {th }}$ decision statistics for $s_{n}^{0}, s_{n}^{1}$ denoted as $z_{n}^{0}(q), z_{n}^{1}(q)$ can be expressed as

$$
\begin{aligned}
z_{n}^{0}(q) & =2 \operatorname{Re}\left\{\left(\mathbf{s}_{q}^{0} \mathbf{h}\right)^{*}\left[\mathbf{r}_{n}-\tilde{\mathbf{S}}_{n}^{0} \mathbf{h}\right]\right\}-\left\|\mathbf{s}_{q}^{0} \mathbf{h}\right\|^{2} \\
& =2 \operatorname{Re}\left\{\left(\mathbf{s}_{q}^{0} \mathbf{h}\right)^{*}\left[\mathbf{S}_{n} \mathbf{h}+\mathbf{w}_{n}-\tilde{\mathbf{S}}_{n}^{0} \mathbf{h}\right]\right\}-\left\|\mathbf{s}_{q}^{0} \mathbf{h}\right\|^{2} \\
& \approx 2 \operatorname{Re}\left\{\left(\mathbf{s}_{q}^{0} \mathbf{h}\right)^{*}\left[\mathbf{s}_{n}^{0} \mathbf{h}+\mathbf{w}_{n}\right]\right\}-\left\|\mathbf{s}_{q}^{0} \mathbf{h}\right\|^{2} \\
& =2 \operatorname{Re}\left\{\left(\mathbf{s}_{q}^{0} \mathbf{h}\right)^{*} \mathbf{s}_{n}^{0} \mathbf{h}\right\}-\left\|\mathbf{s}_{q}^{0} \mathbf{h}\right\|^{2}+\gamma_{q}^{0} ; \\
z_{n}^{1}(q) & =\ldots \approx 2 \operatorname{Re}\left\{\left(\mathbf{s}_{q}^{1} \mathbf{h}\right)^{*} \mathbf{s}_{n}^{1} \mathbf{h}\right\}-\left\|\mathbf{s}_{q}^{1} \mathbf{h}\right\|^{2}+\gamma_{q}^{1} .
\end{aligned}
$$

It is clear to see from the previous equations that the proposed scheme removes the effects of ISI by canceling the contribution of the interfering symbols from the received observation. The approximations in (9) and (10) are owing to the fact that $\mathbf{S}_{n}^{0} \approx \tilde{\mathbf{S}}_{n}^{0}$ and $\mathbf{S}_{n}^{1} \approx \tilde{\mathbf{S}}_{1}^{0}$ as shown in (5). Otherwise, equality would hold in the absence of feedback error, meaning that the interference is completely canceled. The noise terms $\gamma_{q}^{0}=2 \operatorname{Re}\left\{\left(\mathbf{s}_{q}^{0} \mathbf{h}\right)^{*} \mathbf{w}_{n}\right\} \sim \mathcal{C N}\left(0, N_{\gamma}^{0}(q)\right)$, and $\gamma_{q}^{1}=2 \operatorname{Re}\left\{\left(\mathbf{s}_{q}^{1} \mathbf{h}\right)^{*} \mathbf{w}_{n}\right\} \sim \mathcal{C N}\left(0, N_{\gamma}^{1}(q)\right)$. The former can be expanded as

$$
\begin{aligned}
\gamma_{q}^{0}= & 2 \operatorname{Re}\left\{\left(\mathbf{s}_{q}^{0} \mathbf{h}\right)^{*} \mathbf{w}_{n}\right\}=2 \operatorname{Re}\left\{h_{00}^{0 *} s_{q}^{*} w_{n}^{0}+h_{00}^{1 *} s_{q}^{*} w_{n}^{1}\right. \\
& \left.+h_{10}^{0 *} s_{q} w_{n}^{1}+h_{00}^{2 *} s_{q}^{*} w_{n+1}^{0}+h_{10}^{1 *} s_{q} w_{n+1}^{0}+h_{10}^{2 *} s_{q} w_{n+1}^{1}\right\} .
\end{aligned}
$$

Its variance can be derived as

$$
\begin{aligned}
N_{\gamma}^{0}(q) & =\left(\left|h_{00}^{0}\right|^{2}+\left|h_{00}^{1}\right|^{2}+\left|h_{10}^{0}\right|^{2}+h_{00}^{1 *} h_{10}^{0} s_{q}^{*} s_{q}^{*}+h_{00}^{1} h_{10}^{0 *} s_{q} s_{q}\right. \\
& \left.+\left|h_{00}^{2}\right|^{2}+\left|h_{10}^{1}\right|^{2}+\left|h_{10}^{2}\right|^{2}+h_{00}^{2 *} h_{10}^{1} s_{q}^{*} s_{q}^{*}+h_{00}^{2} h_{10}^{1 *} s_{q} s_{q}\right) N_{0} \\
& =\left[P+2 \operatorname{Re}\left\{c_{0} s_{q} s_{q}\right\}\right] N_{0},
\end{aligned}
$$

where $c_{0}=h_{10}^{0 *} h_{00}^{1}+h_{10}^{1 *} h_{00}^{2}$, and $P=\|\mathbf{h}\|^{2}$. The variance of $\gamma_{q}^{1}$ can be derived similarly as

$$
N_{\gamma}^{1}(q)=\left[P-2 \operatorname{Re}\left\{c_{1} s_{q} s_{q}\right\}\right] N_{0},
$$

where $c_{1}=h_{00}^{0 *} h_{10}^{1}+h_{00}^{1 *} h_{10}^{2}$. The first and second terms of $z_{n}^{0}(q)$ in (10) can be derived as

$$
\begin{aligned}
\left(\mathbf{s}_{q}^{0} \mathbf{h}\right)^{*} \mathbf{s}_{n}^{0} \mathbf{h} & =p_{0} s_{q}^{*} s_{n}+p_{1} s_{n}^{*} s_{q}+2 \operatorname{Re}\left\{c_{0} s_{q} s_{n}\right\} ; \\
\left\|\mathbf{s}_{q}^{0} \mathbf{h}\right\|^{2} & =P+2 \operatorname{Re}\left\{c_{0} s_{q} s_{q}\right\},
\end{aligned}
$$

where $p_{0}=\left|h_{00}^{0}\right|^{2}+\left|h_{00}^{1}\right|^{2}+\left|h_{00}^{2}\right|^{2}$, and $p_{1}=\left|h_{10}^{0}\right|^{2}+\left|h_{10}^{1}\right|^{2}+$ $\left|h_{10}^{2}\right|^{2}$. Similarly, the first and second terms of $z_{n}^{1}(q)$ in (10) can be derived as

$$
\begin{aligned}
\left(\mathbf{s}_{q}^{1} \mathbf{h}\right)^{*} \mathbf{s}_{n}^{1} \mathbf{h} & =p_{0} s_{q} s_{n}^{*}+p_{1} s_{q}^{*} s_{n}-2 \operatorname{Re}\left\{c_{1} s_{q} s_{n}\right\} ; \\
\left\|\mathbf{s}_{q}^{1} \mathbf{h}\right\|^{2} & =P-2 \operatorname{Re}\left\{c_{1} s_{q} s_{q}\right\},
\end{aligned}
$$

where $c_{1}=h_{00}^{0 *} h_{10}^{1}+h_{00}^{1 *} h_{10}^{2}$. Based on (13) and (14), we have

$$
\begin{aligned}
& z_{n}^{0}(q) \approx 2 \operatorname{Re}\left\{\left(\mathbf{s}_{q}^{0} \mathbf{h}\right)^{*} \mathbf{s}_{n}^{0} \mathbf{h}\right\}-\left\|\mathbf{s}_{q}^{0} \mathbf{h}\right\|^{2}+\gamma_{q}^{0} \\
& =2 \operatorname{Re}\left\{p_{0} s_{q}^{*} s_{n}+p_{1} s_{n}^{*} s_{q}\right\}+4 \operatorname{Re}\left\{c_{0} s_{q} s_{n}\right\} \\
& \quad-P-2 \operatorname{Re}\left\{c_{0} s_{q} s_{q}\right\}+\gamma_{q}^{0} ; \\
& z_{n}^{1}(q) \approx 2 \operatorname{Re}\left\{\left(\mathbf{s}_{q}^{1} \mathbf{h}\right)^{*} \mathbf{s}_{n}^{1} \mathbf{h}\right\}-\left\|\mathbf{s}_{q}^{1} \mathbf{h}\right\|^{2}+\gamma_{q}^{1} \\
& =2 \operatorname{Re}\left\{p_{0} s_{q} s_{n}^{*}+p_{1} s_{q}^{*} s_{n}\right\}-4 \operatorname{Re}\left\{c_{1} s_{q} s_{n}\right\} \\
& \quad-P+2 \operatorname{Re}\left\{c_{1} s_{q} s_{q}\right\}+\gamma_{q}^{1} .
\end{aligned}
$$

A close examination at equation (15) reveals that the proposed scheme not only preserves the features of the original Alamouti algorithm, i.e, achieving spatial diversity at the receiver, but also takes advantage of the multipath propagation and obtains the temporal diversity by multipath combining (both $p_{0}=\left|h_{00}^{0}\right|^{2}+\left|h_{00}^{1}\right|^{2}+\left|h_{00}^{2}\right|^{2}$, and $p_{1}=\left|h_{10}^{0}\right|^{2}+\left|h_{10}^{1}\right|^{2}+$ $\left|h_{10}^{2}\right|^{2}$ are present in the decision statistics). Based on (15), it can be easily shown that the $m^{\text {th }}$ decision statistics conditioned on the symbol $s_{m}$ being transmitted are

$$
\begin{aligned}
& z_{n}^{0}(m) \mid\left[s_{n}=s_{m}\right] \approx P+2 \operatorname{Re}\left\{c_{0} s_{m} s_{m}\right\}+\gamma_{m}^{0} ; \\
& z_{n}^{1}(m) \mid\left[s_{n}=s_{m}\right] \approx P-2 \operatorname{Re}\left\{c_{1} s_{m} s_{m}\right\}+\gamma_{m}^{1},
\end{aligned}
$$

leading to the conditional PDFs

$$
\begin{aligned}
f\left[z_{n}^{0}(m) \mid s_{m}\right] & =\frac{1}{\pi N_{\gamma}^{0}(m)} \exp \left(-\frac{\left|z_{n}^{0}(m)-P-2 \operatorname{Re}\left\{c_{0} s_{m} s_{m}\right\}\right|^{2}}{N_{\gamma}^{0}(m)}\right) \\
& =\exp \left(\ln \alpha_{0}(m)-\frac{\left|z_{n}^{0}(m)-P-2 \operatorname{Re}\left\{c_{0} s_{m} s_{m}\right\}\right|^{2}}{N_{\gamma}^{0}(m)}\right) ; \\
f\left[z_{n}^{1}(m) \mid s_{m}\right] & =\frac{1}{\pi N_{\gamma}^{1}(m)} \exp \left(-\frac{\left|z_{n}^{1}(m)-P+2 \operatorname{Re}\left\{c_{1} s_{m} s_{m}\right\}\right|^{2}}{N_{\gamma}^{1}(m)}\right) \\
& =\exp \left(\ln \alpha_{1}(m)-\frac{\left|z_{n}^{1}(m)-P+2 \operatorname{Re}\left\{c_{1} s_{m} s_{m}\right\}\right|^{2}}{N_{\gamma}^{1}(m)}\right),
\end{aligned}
$$




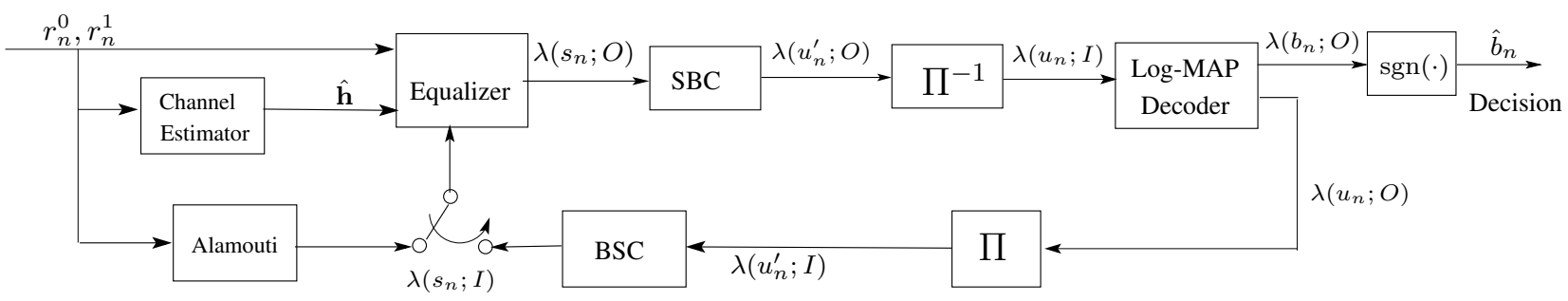

Fig. 2. The proposed turbo equalizer.

where

$N_{\gamma}^{0}(m)=\left[P+2 \operatorname{Re}\left\{c_{0} s_{m} s_{m}\right\}\right] N_{0} ; \quad N_{\gamma}^{1}(m)=\left[P-2 \operatorname{Re}\left\{c_{1} s_{m} s_{m}\right\}\right] N_{0} ;$

$\alpha_{0}(m)=\frac{1}{\pi N_{\gamma}^{0}(m)}=\frac{1}{\pi\left[P+2 \operatorname{Re}\left\{c_{0} s_{m} s_{m}\right\}\right] N_{0}} ;$

$\alpha_{1}(m)=\frac{1}{\pi N_{\gamma}^{1}(m)}=\frac{1}{\pi\left[P-2 \operatorname{Re}\left\{c_{1} s_{m} s_{m}\right\}\right] N_{0}}$.

For QPSK modulated signals, the symbol log-likelihood ratio (LLR) $\lambda\left(s_{n} ; O\right)=\lambda\left(x_{n}\right)+j \lambda\left(y_{n}\right)$ to bits LLRs $\lambda\left(u_{n}^{\prime}[0]\right), \lambda\left(u_{n}^{\prime}[1]\right)$ mapping rule is simply $\lambda\left(u_{n}^{\prime}[0] ; O\right)=$ $\lambda\left(x_{n}\right) ; \quad \lambda\left(u_{n}^{\prime}[1] ; O\right)=\lambda\left(y_{n}\right)$. Next, we show how the LLR value of $x_{n}$ and $y_{n}$ can be derived from $z_{n}^{0}$. Let us define $\max ^{*}(x, y)=\ln \left(e^{x}+e^{y}\right)=\max (x, y)+\ln \left(1+e^{-|x-y|}\right)$, i.e., the max operation compensated with a correction term $\ln \left(1+e^{-|x-y|}\right)$. Based on (16), the LLR value of $x_{n}$ can be computed as

$$
\begin{aligned}
\lambda\left(x_{n}\right) & =\ln \frac{f\left[x_{n}=+1 \mid z_{n}^{0}\right]}{f\left[x_{n}=-1 \mid z_{n}^{0}\right]}-\ln \frac{f\left[x_{n}=+1\right]}{f\left[x_{n}=-1\right]}=\ln \frac{f\left[z_{n}^{0} \mid x_{n}=+1\right]}{f\left[z_{n}^{0} \mid x_{n}=-1\right]} \\
& =\ln \frac{f\left[z_{n}^{0}(0) \mid s_{0}\right]+f\left[z_{n}^{0}(3) \mid s_{3}\right]}{f\left[z_{n}^{0}(1) \mid s_{1}\right]+f\left[z_{n}^{0}(2) \mid s_{2}\right]}=\max ^{*}\left(f_{0}, f_{3}\right)-\max ^{*}\left(f_{1}, f_{2}\right) ; \\
\lambda\left(y_{n}\right) & =\ln \frac{f\left[z_{n}^{0} \mid y_{n}=+1\right]}{f\left[z_{n}^{0} \mid y_{n}=-1\right]}=\ldots=\max ^{*}\left(f_{0}, f_{1}\right)-\max ^{*}\left(f_{2}, f_{3}\right),
\end{aligned}
$$

where

$$
f_{m}=\ln \alpha_{0}(m)-\frac{\left|z_{n}^{0}(m)-P-2 \operatorname{Re}\left\{c_{0} s_{m} s_{m}\right\}\right|^{2}}{N_{\gamma}^{0}(m)} .
$$

The equation (17) holds since the real part of the symbols $s_{0}, s_{3}$ corresponds to 0 , and the real part of the symbols $s_{1}, s_{2}$ corresponds to 1 ; the imaginary part of the symbols $s_{0}, s_{1}$ corresponds to 0 , and the imaginary part of the symbols $s_{2}, s_{3}$ corresponds to 1 .

The LLRs $\lambda\left(u_{n+1}^{\prime}[0]\right), \lambda\left(u_{n+1}^{\prime}[1]\right)$ can be computed similarly based on $z_{n}^{1}$. In this case, $f_{m}$ in (18) should be replaced by

$$
f_{m}=\ln \alpha_{1}(m)-\frac{\left|z_{n}^{1}(m)-P+2 \operatorname{Re}\left\{c_{1} s_{m} s_{m}\right\}\right|^{2}}{N_{\gamma}^{1}(m)},
$$

which is derived based on the definition of $f\left[z_{n}^{1}(m) \mid s_{m}\right]$ in (16). With equations (17), (18) and (19), we can derive the soft output $\lambda\left(u_{n}^{\prime} ; O\right)$ of the equalizer shown in Fig. 2.

\section{Numerical Results}

Numerical results are presented in this section to assess the performance of the proposed turbo equalization scheme. We employ a rate $1 / 3$ Maximum Free Distance convolutional code with constraint length 5 and generator polynomials $(25,33,37)$ in octal form. During each Monte-Carlo run, the block size is set to 2000 information bits followed by 4 tails bits to terminate the trellis, which corresponds to $2004 \times 3=6012$ coded bits or 3006 QPSK symbols, 200 of which are used as pilots to acquire a channel estimate $\hat{h}_{i j}^{l}$. Channel estimation
TABLE I

THE SIMULATED CHANNEL

\begin{tabular}{|c|c|c||}
\hline \hline & Tx0-Rx0 & Tx1-Rx0 \\
\hline$h_{i j}^{0}$ & $0.10+0.14 i$ & $0.046+0.516 i$ \\
\hline$h_{i j}^{1}$ & $-0.19-0.24 i$ & $0.532+0.14 i$ \\
\hline$h_{i j}^{2}$ & $0.472+0.15 i$ & $0.25-0.34 i$ \\
\hline
\end{tabular}

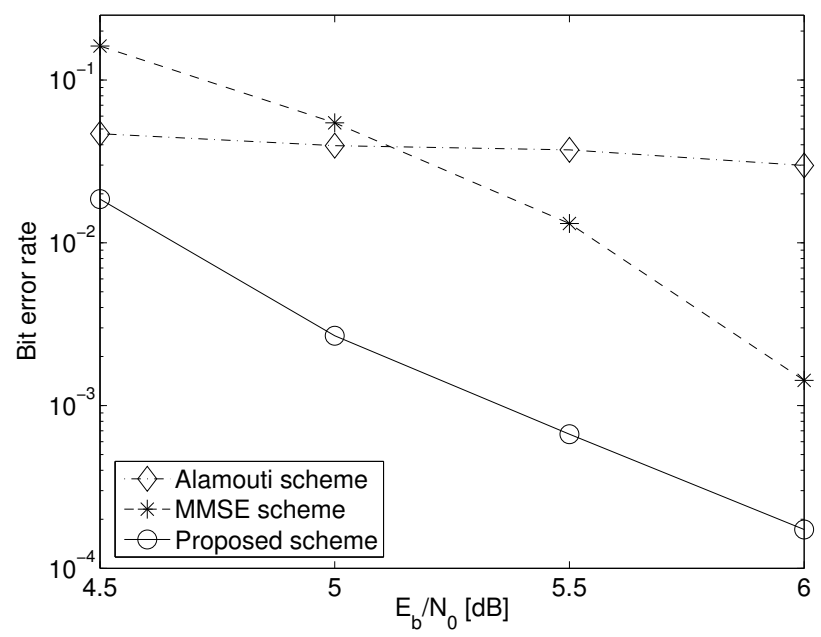

Fig. 3. Comparison of different schemes.

is conducted with the modified maximum likelihood algorithm introduced in [8]. The coded bits are interleaved by a random interleaver. The noise variance $N_{0}$ and path delays are assumed to be known to the receiver. The simulation curves are obtained by averaging the simulation results over a minimum of 30 blocks of data transmitted and after at least 100 errors are generated. The Alamouti algorithm is used to obtain an initial estimate of the transmitted symbols. The basic principle is to apply the Alamouti detection scheme on each path and combine the desired signals from different paths to obtain multipath diversity gain.

In our simulations, we tested a $2 \mathrm{Tx}-1 \mathrm{Rx}$ channel with complex channel gains specified in Table I (before normalization). The channel coefficients are normalized such that $\sum\left|h_{i, j}^{l}\right|^{2}=$ 1.0 , where $j=0$, and the summation is carried out over all possible values of $i \in\{0,1\}$ and $l \in\{0,1,2\}$. Fig. 3 shows the comparison of different schemes. As expected, the proposed turbo equalizer outperforms the MMSE based scheme. For example, at BER of $10^{-2}$, the performance penalty by the MMSE scheme is around $1 \mathrm{~dB}$ compared to the proposed scheme. The reason is that the proposed scheme not only effectively reduces the effects of ISI by canceling the interference as shown by equation (10), but also achieves spatial and temporal diversity 
TABLE II

\begin{tabular}{|c|c|c|c|}
\hline \hline operations & $*$ & $/$ & $+/-$ \\
\hline Proposed scheme & $8 L+17$ & 4 & $8 L+23$ \\
\hline MMSE scheme & $64 L^{2}+80 L+173$ & 36 & $64 L^{2}+64 L+145$ \\
\hline
\end{tabular}

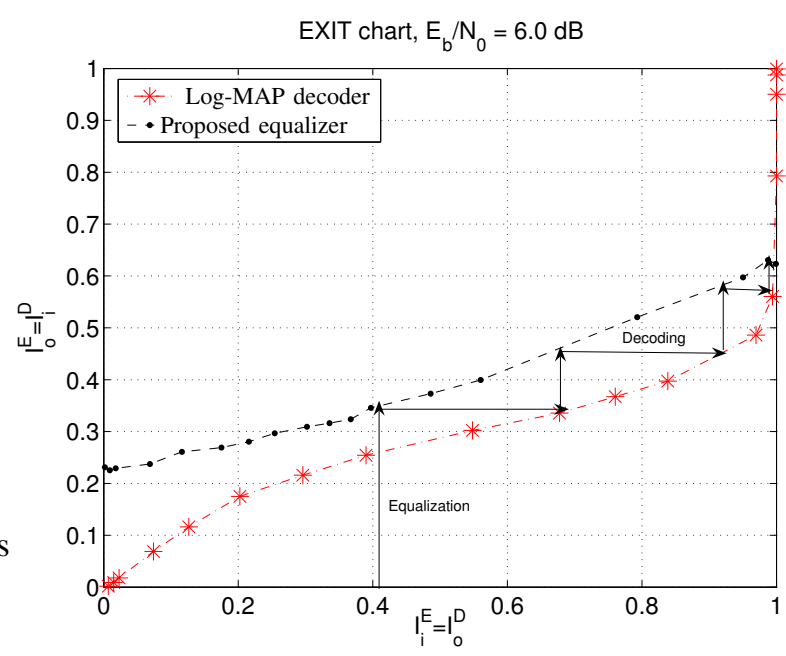

Fig. 4. EXIT chart analysis of the proposed scheme at $E_{b} / N_{0}=6.0 \mathrm{~dB}$.

as shown by equation (15). On the contrary, the MMSE scheme cannot utilize the spatial diversity as discussed in [9]. To work around this problem, the solution in [5] is not to use any STBC or STTC. The curve of the original Alamouti algorithm is also plotted in the figure and is shown to be inferior to all the schemes at high $E_{b} / N_{0}$ when ISI is dominant. The reason is that the Alamouti algorithm does not take the ISI into account, and thus suffers the most from the detrimental effects of ISI; whereas other schemes effectively remove the ISI by canceling interference with decision feedback. However, at low $E_{b} / N_{0}$, the original Alamouti algorithm shows better performance than the MMSE scheme because the ISI is not effectively canceled at low $E_{b} / N_{0}$ when the noise is dominant. In this case, the turbo equalization schemes suffer from the problem of error propagation.

The proposed scheme only require linear processing as the original Alamouti algorithm. Table II shows the required number of complex multiplications (*), divisions (/), and additions/subtractions $(+/-)$ for each QPSK symbol estimate by different turbo equalization schemes. $L$ is the number of channel taps. Apparently, the proposed scheme reduces the complexity from $O\left(L^{2}\right)$ to $O(L)$ compared to the MMSE based turbo equalization scheme. As the number of multipath components increases, the complexity reduction becomes more significant.

The convergence behavior of the proposed scheme is investigated at $E_{b} / N_{0}=6 \mathrm{~dB}$ in Fig. 4 using the extrinsic information transfer (EXIT) chart which traces the evolution of the mutual information $I_{i}^{E} / I_{o}^{E} \in[0,1]$ between input/output LLR and $s_{n}$ for equalization; and the mutual information $I_{i}^{D} / I_{o}^{D}$ between input/output LLR and $u_{n}$ for decoding. Refer to [10] for detailed discussion of this analysis method and [4] for its application in turbo equalization.

It should be noted that in our case, $I_{i}^{E}>0$ at the beginning of the iterative process. This is due to the presence of the initial Alamouti detection stage (see the lower left corner in Fig. 2). Therefore, the a priori information is not zero when the turbo equalization starts. In Fig. 4, the iterative equalization and decoding process is depicted by a staircase trace between the transfer curves of the equalizer and decoder $(\rightarrow$ represents the decoding process and $\uparrow$ represents the equalization process). The expected trace is shown in this figure. The real trace slightly deviates the predicted one due to the finite interleaver length. However, the discrepancy is small. The trace shows that only 4 stages of equalization/decoding are needed for the proposed scheme to converge (reach the maximum $I_{o}^{D}$ ) at $E_{b} / N_{0}=6 \mathrm{~dB}$. The majority of the gain is obtained at the first and second iterations. (Note that there is a decoding step - the fourth one at the end of the trace which is hardly visible as the diagram gets too cramped.) This is in close agreement with our simulation results.

\section{CONCLUSIONS}

The use of multiple antennas in combination with advanced detection techniques, such as turbo equalization is an effective means for a communication system to provide high quality and high data rate services. Alamouti's space-time block code with two transmit antennas and one or two receive antennas over frequency selective MIMO channel is considered in this paper. In order to overcome the prohibitive computational complexity imposed by the maximum likelihood detection for the system under investigation, we propose a low complexity detection scheme, which is shown to provide large performance gains compared to the original Alamouti algorithm and the conventional linear MMSE equalization for the STBC coded systems over frequency selective channels.

\section{ACKNOWLEDGEMENT}

This work was sponsored by the UK Engineering and Physical Sciences Research Council under grant number EP/D07827X/1. The authors would like to acknowledge its financial support.

\section{REFERENCES}

[1] V. Tarokh, N. Seshadri, A. Calderbank. "Space-time codes for high data rates wireless communications: performance criterion and code construction". IEEE Transactions on Information Theory, vol. 44, pp. 744-765, March 1998.

[2] A. Alamouti. "A simple transmit diversity technqiue for wireless communications". IEEE Journal on Selected Areas in Communications, vol. 16, no. 8, pp. 1451-1458, Oct. 1998.

[3] X. Wang, H. Poor. "Iterative (turbo) soft interference cancellation and decoding for coded CDMA". IEEE Transactions on Communications, vol. 47, pp. 1046-1061, July 1999.

[4] M. Tuchler, R. Koetter, A. Singer. "Turbo equalization: principles and new results". IEEE Transactions on Communications, vol. 50, pp. 754767, May 2002.

[5] X. Wautelet, A. Dejonghe, L. Vandendorpe. "MMSE-based fractional turbo receiver for space-time BICM over frequency-selective MIMO fading channels". IEEE Transactions on Signal Processing, vol. 52, no. 6, pp. 1804-1809, June 2004.

[6] T. Abe, T. Matsumoto. "Space-time turbo equalization in frequencyselective MIMO channels". IEEE Transactions on Vehicular Technology, vol. 52, no. 3, pp. 469-482, May 2003.

[7] B. Lu and X. Wang. "Iterative receivers for multiuser space-time coding systems". IEEE Journals on Selected Areas in Communications, vol. 18, no. 11. pp. 2322-2335.

[8] P. Xiao, R. Carrasco, I. Wassell. "Estimation of FWA MIMO channels". In Proc. IEEE Information Theory Workshop, pp. 641-645, Oct. 2006.

[9] P. Xiao, R. Carrasco, I. Wassell. "Turbo equalization for space-time block coded FWA systems". In Proc. IEEE Information Theory Workshop, pp. 626-630, Oct. 2006.

[10] S. Brink. "Convergence behavior of iterative decoded parallel concatenated codes". IEEE Transactions on Communications, vol. 49, no. 10, pp. 1727-1737, Oct. 2001. 